\title{
Trauma unit workload at King Edward VIII Hospital, Durban, KwaZulu-Natal
}

K K Pillay, A Ross, S van der Linde

Each year, 70000 South Africans are killed by trauma, and a further 3.5 million seek trauma care. We analysed 1465 trauma patients at the regional-level King Edward VIII Hospital (KE8H) in eThekwini Municipality. Mean patient age was 29 years. Peaks in patient numbers were observed mid-month, at month-end, between $08 \mathrm{~h} 00$ and $12 \mathrm{~h} 00$ daily and on Saturday and Sunday nights. Most injuries occurred on roads, at informal settlements and at bars/shebeens. More than $44 \%$ of injuries had a violent cause. The pattern of presentation was more in line with a primary healthcare setting than a referral centre. Reliable and accurate injury trend information is required for effective strategies to curb South Africa's high injury mortality and morbidity rates. Epidemiological databases are needed, as well as safe, robust and ethical systems for collecting, collating, analysing and disseminating non-fatal injuryrelated data.

S Afr Med J 2012;102(5):307-308
Deficiencies in trauma care are a worldwide problem owing to the difficulty of assessing the burden of non-fatal injury. Mortality statistics are used as indicators of violence and injury; however, nonfatal injuries exceed fatal injuries by up to 20 times. ${ }^{1}$ Non-fatal injury data in Africa rely on limited studies and population groups, resulting in a 'patchwork picture of the injury profile of Africa.' ${ }^{1}$ The lack of reliable statistics makes it difficult to assess the impact of societal changes on the rate of injuries or to compare South Africa with other settings. An assessment of the effect of non-fatal outcomes on the overall injury burden is needed.

We studied the workload at a leading trauma unit (TU) in terms of temporal distribution of work, severity of workload by triage category and injury types.

\section{Methods}

The study was conducted at the TU of King Edward VIII Hospital (KE8H), eThekwini Municipality, in August 2011. A data-capture form and time sheet were completed for every trauma patient seen in the unit over the study period. Approval was obtained from the Biomedical Research Ethics Committee at the University of KwaZulu-Natal.

\section{Results}

A total of 2037 patients were examined, including 572 (28\%) nontrauma and 1465 (72\%) trauma patients respectively. This translates to 17500 trauma patients annually. Mean patient age was 29.47 years, and $63 \%$ of patients were males aged 18 - 35 years. Classification of

Department of Family Medicine, Nelson R Mandela School of Medicine, University of KwaZulu-Natal, and Trauma Unit, King Edward VIII Hospital, Durban K K Pillay, BSc (Hons), MB BCh

Department of Family Medicine, Nelson R Mandela School of Medicine, University of KwaZulu-Natal

A Ross, MB ChB, DCh, MFamMed (MEDUNSA)

Department of Public Health, Programme for Bioethics and Biostatistics, College of Health Sciences, University of KwaZulu-Natal

$S$ van der Linde, MSC the workload included $49 \%$ green-code and $7 \%$ red-code injuries (Fig. 1). The trauma workload showed a bimodal peak, at mid-month and month-end weekends. There was no significant variation in dayto-day non-trauma workload.

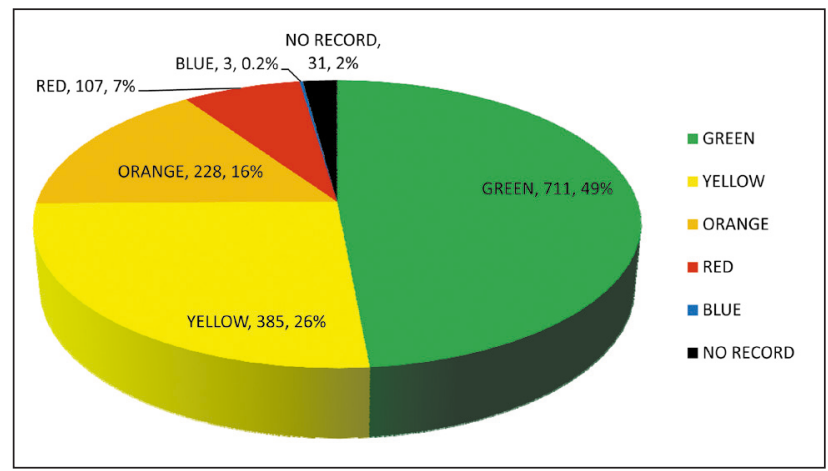

Fig. 1. Distribution of trauma workload per triage category.

Injuries were presented as follows: $32.6 \%$ between $08 \mathrm{~h} 00$ and $12 \mathrm{~h} 00$, including $56.7 \%$ of green-code patients; $33.4 \%$ over the weekend, of which $24.7 \%$ were red- or orange-code; and $31.1 \%$ at night $(20 \mathrm{~h} 00-08 \mathrm{~h} 00)$, of which $11.4 \%$ were red-code (compared with $5.4 \%$ during the day). There were 107 red-code injuries; $41.1 \%$ were seen over the weekend and $48.6 \%$ at night (Fig. 2). Workload over the 4 weekends of the study included 490 patients; 201 were seen between $20 \mathrm{~h} 00$ and $08 \mathrm{~h} 00$, and $9 \%$ had red-code injuries. The lowest number of patients was seen between $16 \mathrm{~h} 00$ and $20 \mathrm{~h} 00$ on Tuesdays and Thursdays.

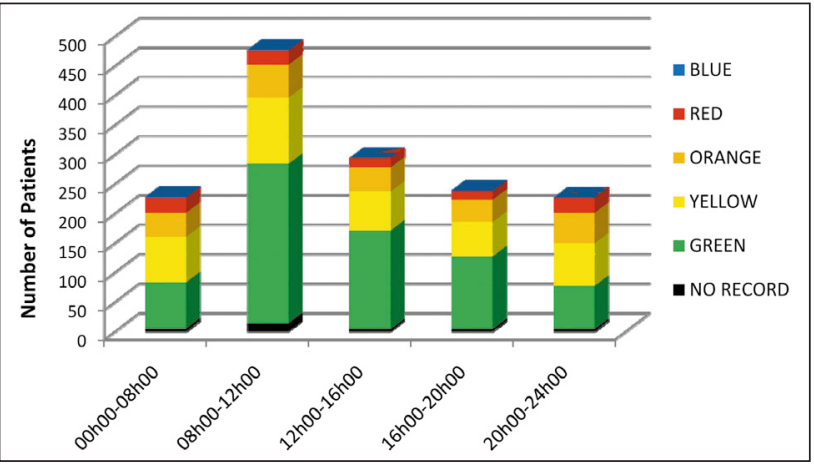

Fig. 2. Temporal distribution of workload per triage category. 
Violent and accidental injuries amounted to $38.9 \%$ and $38.6 \%$, respectively, with $67.3 \%$ of all red-code injuries and $55.7 \%$ of all orange-code injuries attributable to a violent cause. Violent injuries accounted for $34.9 \%$ and $31.9 \%$ of serious and green-code injuries, respectively. Transport-related and self-inflicted intentional injuries accounted for $21.5 \%$ and $0.8 \%$ of the total injuries, correspondingly. The injuries of 31 patients were not classified (Fig. 3).

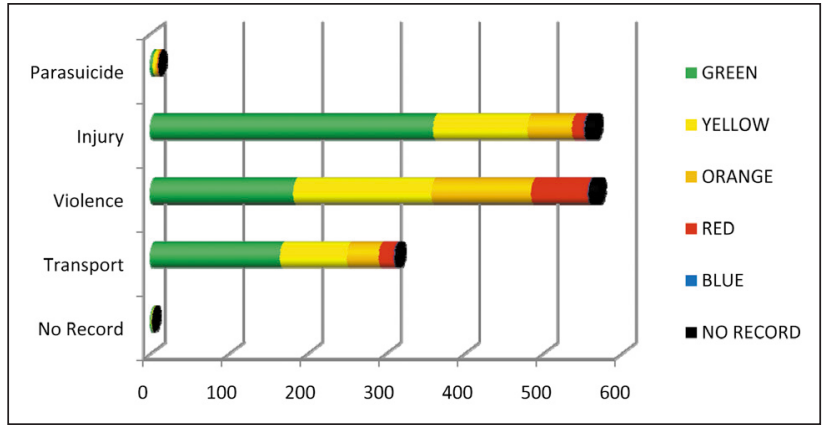

Fig. 3. Injury type according to triage category.

\section{Waiting times}

Waiting times of 1085 of the 1465 patients were recorded. Greencode patients were examined within 60 minutes of arrival. Irrespective of triage category, trauma patients spent approximately 280 minutes in the unit and waited about 40 minutes before examination by the casualty doctor. After consultation, patients waited $>90$ minutes on average for review of their laboratory or radiological results. Redcode patient radiological investigations were performed and reviewed in less than an hour. There was no significant difference in waiting times on different weekdays.

\section{Discussion}

TU workload impacts on resource planning and organisation. In a similar 4 -week period in 2000, 832 trauma patients were seen, compared with the 1465 in this study. ${ }^{2}$

The estimated annual trauma patient load ( $>17500)$ is comparable with that of the leading level-one TU in Gauteng (16 357). ${ }^{3}$ These units have similar demographic workloads with predominant age groups of 18 - 35 and 16 - 35 years, respectively. ${ }^{3}$ Of the 5399 deaths recorded in eThekwini in 2007, the majority (1 310) were in the age group of 15 - 34 years. ${ }^{4}$ This group represents a significant proportion of the work force, highlighting the socio-economic consequences of trauma injuries.

The triage category percentages were more representative of that of a primary healthcare facility than of a referral centre. KE8H was expected to have a higher proportion of serious cases. For every red-code patient, there were 2.3 orange-code, 3.7 yellow-code and 7 green-code patients, respectively. This may have been attributed to self-referral by patients with minor injuries. A prior study in Cape Town showed that $60.4 \%$ of patients sought 'inappropriate trauma care. $^{.5}$

Only 3 deaths ( $0.2 \%$ mortality rate) were recorded in the month of the study, compared with $0.6 \%$ in Cape Town ${ }^{6}$ and $1.4 \%$ in Johannesburg. ${ }^{3}$ The 2007 injury mortality rate for eThekwini was $134.8 / 100000(0.13 \%){ }^{4}$

The South African Triage Scale (SATS) provides time guidelines for the assessment of patients classified in different triage categories. Only $74.4 \%$ of red-code patients were examined within 15 minutes of triage (average time interval of 12.8 minutes). This is unacceptable in accordance with the SATS performance threshold indicator: 95\% of red-code patients should be seen almost immediately after triage. ${ }^{7}$
Orange-code patients should be examined within 10 minutes of triage (SATS performance threshold of $80 \%) .^{7}$ In this study, such patients were examined within 18 minutes; only $32.8 \%$ were seen within 10 minutes. Yellow- and green-code patients were examined within 26 minutes of triage, and an average 30 minutes after triage, respectively. SATS advocates a maximum of 60 minutes (75\% performance threshold indicator) for yellow-code patients and a maximum of 240 minutes (70\% performance threshold indicator) for green-code patients.

The unit operated within SATS guidelines for yellow- and greencode patients, but in serious cases there was a delay between the time of triage and assessment. Reasons for this included: (i) the resuscitation of seriously injured patients by trauma-trained nurses before the casualty doctor commenced management; (ii) the presence of more than one serious case in the unit at a time; and (iii) a shortage of medical and nursing staff. On average, patients spent almost 5 hours in the TU, even for minor injuries. In the UK, $98 \%$ of accident and emergency unit patients spend $<4$ hours from the time of arrival to leaving the unit. ${ }^{8}$

The long waiting times for the review of laboratory and radiological investigation results appeared to be the main reason for delays. Waiting times could have been reduced by: (i) point-of-care testing/satellite laboratories as opposed to centralised laboratory investigations; (ii) provision of a dedicated 24-hour pharmacy for trauma patients; (iii) keeping incorrect referrals to a minimum; (iv) patient education; and $(v)$ primary care gate-keeping. However, the safety of potentially delaying the tertiary management of life-threatening emergencies has not been studied in such a setting. Earlier assessment of patients by senior staff could also significantly reduce waiting times; this is difficult to implement in an academic setting.

Globally, injuries cause 5 million deaths per annum or 16000 deaths per day. Five of the 15 leading causes of death in persons aged 15 - 29 years are unintentional injury-related. ${ }^{9}$ Deaths are a small proportion of the injury burden. Non-fatal health outcomes represent a larger component, with a substantial number of injuries resulting in potentially life-long disability, significant psychological trauma and financial loss.

Acknowledgements. We thank Dr Sailuja Naidu for support, counsel and manuscript proof-reading, Dr S A Moodley (Acting Medical Manager, KE8H), Mrs P Bhadree (Assistant Pharmacy Manager, MOPD Pharmacy, KE8H), full- and part-time doctors and nursing and clerical staff at KE8H TU, Mr Xaba (Health Research \& Knowledge Management Subcomponent, Department of Health, KZN), Mr C Naidu and Mr K R Pillay for assistance with data collection and processing.

\section{References}

1. World Health Organization (WHO). World Report on Violence and Health. Geneva: WHO, 2002.

2. Peden M, Olin A, Sukhai A, Harris, C. National Non-Fatal Injury Surveillance System, King Edward VIII hospital pilot study report. Department of Arts, Culture, Science and Technology, 2000:1-14. VIII hospital pilot study report. Department of Arts, Culture, Science and Technology, 2000:1-14.
3. Bruce JC, Schmollgruber S, Eales, J, Gassiep J, Doubell V. Injury surveillance at a level I trauma centre 3. Bruce JC, Schmollgruber S, Eales, J, Gassiep J, Doubell V. Injury survei
in Johannesburg, South Africa. Health SA Gesondheid 2003;8(3):3-12.

4. Neethling I. A profile of fatal injuries in eThekwini (Durban). In: Donson H, ed. A Profile of Fatal Injuries in South Africa. Tygerberg: MRC-UNISA Crime, Violence and Injury Lead Programme, 2007. 5. Peden $M$, van der Spuy J, Abrahams, N. The inappropriate use of tertiary trauma facilities. Trauma and Emergency Medicine 1996;13(1):20-21.

6. Wallis LA, Towney M. Workload and casemix in Cape Town emergency departments. S Afr Med J 2007;97(12):1276-1280.

Twomey M. Implementation of the South African Triage Scale, Emergency Medicine Society of South Africa Practice guideline EM014. http://emssa.org.za/documents/em014.pdf (accessed 23 June 2010). Department of Health (UK). Towards faster treatment: reducing attendance and waits at emergency Department of Health (UK). Towards faster treatment: reducing attendance and waits at emergency departments. Emergency Care and Rehabilitation. NHS Service Delivery and Organisation R\&D
Programme Briefing Paper. London, UK: Department of Health, 2005 . 9. World Health Organization (WHO). Violence, Injuries, and Disability: Biennial 2006-2007 Report. Geneva: WHO, 2008

Accepted 1 March 2012. 